\title{
Effect of Antenna Array Element Separation on Capacity of MIMO Systems Including Mutual Coupling
}

\author{
Tomislav Marinovic ${ }^{* * \dagger \S}$, Amirashkhan Farsaei ${ }^{\ddagger \S}$, Rob Maaskant ${ }^{\dagger \ddagger}$, Adrián Lahuerta Lavieja*£, \\ Martin N. Johansson ${ }^{\S}$, Ulf Gustavsson ${ }^{\S}$, Guy A.E. Vandenbosch* \\ * ESAT-TELEMIC, KU Leuven, Leuven, Belgium \\ $\dagger$ Dept. of Electrical Engineering, Chalmers University of Technology, Gothenburg, Sweden \\ $\ddagger$ Dept. of Electrical Engineering, TU Eindhoven, Eindhoven, The Netherlands \\ $\S$ Ericsson Research, Gothenburg, Sweden
}

\begin{abstract}
In this paper, we study the effect of element separation in a base station array (BSA) on the downlink line-of-sight achievable sum-rate of MIMO systems in the presence of mutual coupling at the BSA. The channel matrix $H$ is modeled using the embedded field patterns of the BSA and is used to analyze the outage performance for two single-element user equipments assuming no inter-user coupling.
\end{abstract}

\section{INTRODUCTION}

The effect of base station array (BSA) antenna element separation on the capacity of point-to-point MIMO systems, assuming a Rayleigh channel and including mutual coupling (MC) at transmitter and receiver, has been studied in [1], [2]. Similarly, MC has been shown to have a beneficial effect on the capacity of multi-user MIMO for spacings closer than halfwavelength in a Rayleigh environment [3]. In a line-of-sight (LOS) environment, MC effects have been studied in [4] for a fixed array spacing. This paper presents a study of the effect of inter-element spacing on the achievable sum-rate of a LOS MIMO system with two users and a BSA modeled with MC.

\section{Methodology}

\section{A. Network representation of the system}

Fig. 1 shows a network representation of an entire MIMO system with $M$ BSA antennas serving $K$ users [1]. The

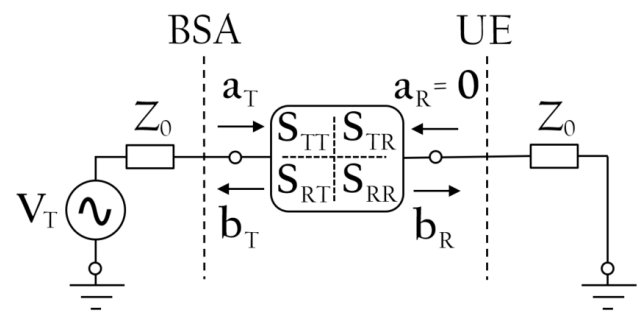

Fig. 1: Block-based network model of a MIMO system.

transmit array is driven through a Thévenin voltage source equivalent with an RMS source voltage $V_{\mathrm{T}}$ and a complex source impedance $Z_{0}$ as shown in Fig 1. A block-partitioned matrix equation of the system is given as [1]

$$
\left[\begin{array}{l}
\boldsymbol{b}_{\mathrm{T}} \\
\boldsymbol{b}_{\mathrm{R}}
\end{array}\right]=\left[\begin{array}{ll}
\boldsymbol{S}_{\mathrm{TT}} & \boldsymbol{S}_{\mathrm{TR}} \\
\boldsymbol{S}_{\mathrm{RT}} & \boldsymbol{S}_{\mathrm{RR}}
\end{array}\right]\left[\begin{array}{c}
\boldsymbol{a}_{\mathrm{T}} \\
\boldsymbol{a}_{\mathrm{R}}=\mathbf{0}
\end{array}\right],
$$

where $\boldsymbol{S}_{\mathrm{TT}}$ and $\boldsymbol{S}_{\mathrm{RR}}$ are the scattering matrices of the transmit and receive arrays, and $\boldsymbol{S}_{\mathrm{RT}}$ and $\boldsymbol{S}_{\mathrm{TR}}$ represent the BSAUE and UE-BSA channel matrices, respectively. Furthermore, $\boldsymbol{a}$ and $\boldsymbol{b}$ are defined as incident and reflected block-based power wave vectors, respectively. Assuming that the receiver is perfectly matched, $\boldsymbol{a}_{\mathrm{R}}=\mathbf{0}$. To represent the channel matrix $\boldsymbol{S}_{\mathrm{RT}} \in \mathcal{C}^{K \times M}$, we use the embedded field patterns $\boldsymbol{E}^{\mathrm{emb}}$ of the transmit antennas generated using the Thévenin voltage source. It follows from (1) that the power wave $b_{R_{k}}$ is proportional to both $\boldsymbol{E}^{\mathrm{emb}}$ and $a_{\mathrm{T}}$ as [5, Ch. 2]

$$
\left|b_{\mathrm{R}_{k}}\right| \propto\left|\hat{\boldsymbol{l}}_{k} \cdot \sum_{n=1}^{N_{\mathrm{T}}} a_{n}^{T} \boldsymbol{E}_{n}^{\mathrm{emb}}\left(r_{k}, \phi_{k}\right)\right|,
$$

where the relation $a_{\mathrm{T}}=V_{\mathrm{T}} /\left(2 \sqrt{\mathcal{R}\left\{Z_{0}\right\}}\right)$ [6, Ch. 2], and with $\hat{\boldsymbol{l}}_{k}$, and $\left(r_{k}, \phi_{k}\right)$ being the polarization vector and polar coordinates of user $k$, respectively.

\section{B. Capacity}

To model the downlink case, we use the channel-noise model proposed by [1, sec. 4.3.]. The relation between the power wave vector $\boldsymbol{b}_{\mathrm{R}} \in \mathcal{C}^{K \times 1}$ and the excitation power wave vector $\boldsymbol{a}_{\mathrm{T}} \in \mathcal{C}^{M \times 1}$ at the BSA is given as

$$
\boldsymbol{b}_{\mathrm{R}}=\left(\boldsymbol{S}_{\mathrm{RT}} \boldsymbol{a}_{\mathrm{T}}+2 \mathcal{R}\left\{Z_{0}\right\}^{-1 / 2} \boldsymbol{n}\right),
$$

where $n \in \mathcal{C}^{K \times 1}$ is the AWGN vector. The total radiated power constraint is chosen according to (9) of [1]. Therefore, the downlink capacity with total radiated power $P_{\text {tot }}$ is

$$
\begin{array}{r}
C=\max _{\boldsymbol{K}_{a_{\mathrm{T}}}} \log _{2}\left[\operatorname{det}\left(\boldsymbol{I}_{K}+\frac{1}{N_{0}} \boldsymbol{H} \boldsymbol{K}_{a_{\mathrm{T}}} \boldsymbol{H}^{H}\right)\right], \\
\text { s.t. } \operatorname{trace}\left(\boldsymbol{K}_{a_{\mathrm{T}}}\left(\boldsymbol{I}_{M}-\boldsymbol{S}_{\mathrm{TT}}^{H} \boldsymbol{S}_{\mathrm{TT}}\right)\right) \leq P_{\text {tot }},
\end{array}
$$


where $\boldsymbol{K}_{a_{\mathrm{T}}}$ is the covariance matrix of $\boldsymbol{a}_{\mathrm{T}}, \boldsymbol{H}=\boldsymbol{S}_{\mathrm{RT}}, N_{0}$ is the noise power at a user receiver, and $\boldsymbol{I}_{K}$ and $\boldsymbol{I}_{M}$ are identity matrices of size $K$ and $M$, respectively. Note that $P_{\text {tot }}$ is found by an appropriate EIRP analysis to ensure a certain SNR at the users. It is assumed that only the BSA knows the downlink channel. By choosing equal power allocation among the BSA antennas $\boldsymbol{K}_{a_{\mathrm{T}}}^{*}=\frac{P_{\mathrm{tot}}}{\operatorname{trace}\left(\boldsymbol{I}_{M}-\boldsymbol{S}_{\mathrm{TT}}^{H} \boldsymbol{S}_{\mathrm{TT}}\right)} \boldsymbol{I}_{M}[7$, Ch. 8], the following achievable sum-rate is found:

$$
R=\log _{2}\left[\operatorname{det}\left(\boldsymbol{I}_{K}+\frac{1}{N_{0}} \boldsymbol{S}_{\mathrm{RT}} \boldsymbol{K}_{a_{\mathrm{T}}}^{*} \boldsymbol{S}_{\mathrm{RT}}^{H}\right)\right] .
$$

\section{NumericAl Results}

We study a BSA consisting of a $4 \times 1$ uniform linear array (ULA) of center-fed half-wavelength $z$-oriented strip dipoles distributed along the $x$-axis. The BSA is serving $K=2$ co-polarized single-antenna UEs. Inter-user MC is neglected. Both the BSA and UEs are assumed perfectly matched. The users are uniformly distributed in the $H$-plane far-field of the $\mathrm{BSA}$ at a radius of $100 \mathrm{~m}$. The field-of-view (FoV) is chosen from $\phi=30^{\circ}$ to $\phi=150^{\circ}$ with $1^{\circ}$ sampling. The following procedure is applied to calculate the 5\% outage for different BSA inter-element spacings in the range of $0.01 \lambda$ to $2 \lambda$ :

1) Generate $\boldsymbol{S}_{\mathrm{TT}}$ and the channel matrix $\boldsymbol{H}=\boldsymbol{E}^{\mathrm{emb}}$ for a given array geometry. For this we use the 'CAESAR' EM solver [8]. The BSA is driven by a Thévenin source with $V_{\mathrm{T}}=1 \mathrm{~V}$ and $Z_{0}=50 \Omega$.

2) Find achievable sum-rates using (5) for all possible combinations of user locations within the FoV $\left(\phi_{k=1}, \phi_{k=2}\right)$. After discarding the lowest $5 \%$ values of the achievable sumrate, the minimum $\left(R_{\min }\right)$, average $\left(R_{\mathrm{avg}}\right)$, and maximum $\left(R_{\max }\right)$ sum-rates are found and plotted in Fig. 2. For comparison, the sum-rates calculated by assuming minimum-scattering antennas (MSA, $Z_{0} \rightarrow \infty$, open-circuited dipoles) at the BSA are plotted in the same figure. When the UE element spacing approaches zero, it becomes increasingly more difficult to distinguish between the two users in space. This causes a drop in $R_{\max }$ and $R_{\text {avg }}$ to the value of $R_{\min }$. Mutual coupling has a beneficial impact on the maximum sum-rate for all element spacings compared to the MSA model. On the other hand, it causes a detrimental effect on the minimum sum-rate for spacings closer than $1 \lambda$. In terms of the average sumrate, $\lambda / 2$ spacing offers the best performance. As expected, with increasing wavelength and decaying coupling effects, the curves including MC converge to the MSA model.

\section{CONCLUSION}

In this paper we have studied the effect of BSA element spacing on the achievable sum-rate of a LOS MIMO system. Numerical results are presented for a system consisting of a $4 \times 1$ transmit ULA and two randomly distributed co-polarized single-antenna users. To account for the coupling effects at the transmit array, embedded element patterns have been used to model the channel matrix $\boldsymbol{H}$. It has been demonstrated that the MC has positive impact on the maximum sum-rate regardless of inter-element spacing, and negative impact on

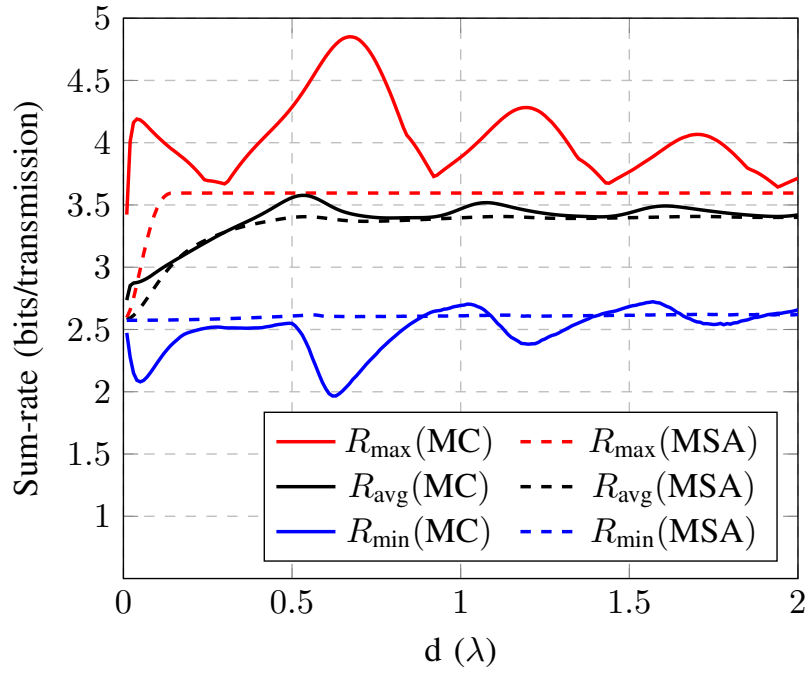

Fig. 2: Sum-rates $R_{\min }, R_{\text {avg }}$ and $R_{\max }$ considering mutual coupling (MC) or assuming minimum-scattering antennas (MSA) at the BSA.

the minimum sum-rate for spacings less than $1 \lambda$. This is in contrast with the often-used assumption that the MC has a detrimental effect on the system performance for any given array geometry. The effects of different array geometries and channel considerations will be addressed in a future study.

\section{ACKNOWLEDGMENT}

This project has received funding from the European Union's Horizon 2020 research and innovation program under the Marie Skłodowska-Curie grant agreement No. 721732.

\section{REFERENCES}

[1] J. W. Wallace and M. A. Jensen, "The capacity of MIMO wireless systems with mutual coupling," in Proceedings IEEE 56th Vehicular Technology Conference, vol. 2, Sep. 2002, pp. 696-700 vol.2.

[2] - "Mutual coupling in MIMO wireless systems: a rigorous network theory analysis," IEEE Trans. Wireless Commun., vol. 3, no. 4, pp. 13171325, July 2004.

[3] F. Wang and M. E. Bialkowski, "Performance of successive broadcasting scheme for a multiuser dowlink MIMO system operating under mutual coupling conditions," in 2010 4th International Conference on Signal Processing and Communication Systems, Dec 2010, pp. 1-4.

[4] C. Chen, V. Volski, L. V. der Perre, G. A. E. Vandenbosch, and S. Pollin, "Finite large antenna arrays for massive mimo: Characterization and system impact," IEEE Trans. Antennas Propag., vol. 65, no. 12, pp. 6712 6720, Dec 2017.

[5] P.-S. Kildal, Foundations of Antenna Engineering: A Unified Approach for Line-of-Sight and Multipath. Gothenburg: Kildal Antenn AB, 2015.

[6] J. A. Dobrowolski, Introduction to Computer Methods for Microwave Circuit Analysis and Design. Warsaw University of Technology: Artech House, 1991.

[7] D. Tse and P. Viswanath, Fundamentals of Wireless Communication. New York, NY, USA: Cambridge University Press, 2005.

[8] R. Maaskant, "Analysis of large antenna systems," Ph.D. dissertation, Eindhoven University of Technology, Eindhoven, 2010. [Online]. Available: http://alexandria.tue.nl/extra2/201010409.pdf 